\title{
ADAPTACIÓN TRANSCULTURAL DE MENSAJES DE TEXTO PARA AUTOCUIDADO EN GESTANTES
}

Consuelo Vélez-Álvarez' ${ }^{1}$ Claudia Patricia Jaramillo-Ángel², Jorge Alejandro García-Ramírez ${ }^{3}$, Camilo Barrera-Valencia ${ }^{4}$

\section{Resumen}

El objetivo de este trabajo fue adaptar transculturalmente mensajes de texto dirigidos a gestantes y sus acompañantes para dar herramientas a la academia en los procesos educativos. Se realizó adaptación transcultural en 4 fases: la primera partió de la revisión de textos traducidos al español por los docentes investigadores; luego se procedió a la revisión por parte de expertos; la tercera fase se articuló a partir de grupos focales con gestantes y acompañantes, y finalmente la elaboración de nuevos mensajes de acuerdo con guías y normas de atención en salud. En total, fueron revisados 170 mensajes en la primera fase: los expertos revisaron 107 dirigidos a gestantes, 37 a acompañantes, 18 sobre manejo de recién nacido, sobre estos se hicieron ajustes. El resultado de los grupos focales consolidó el proceso de claridad de los mensajes. El artículo propone una metodología para la adaptación transcultural que permite a la academia obtener mensajes que las gestantes requieren para fortalecer su autocuidado.

Palabras clave: metodología, educación, embarazo, mensaje de texto, tecnología educacional.

${ }^{1}$ Doctora en Salud Pública. Docente Universidad de Caldas, Manizales, Colombia. Correo electrónico: consuelo.velez@ucaldas.edu.co

${ }^{2}$ Doctora en Salud Pública. Docente Universidad de Caldas, Manizales, Colombia.

${ }^{3}$ Médico. Docente Universidad de Caldas, Manizales, Colombia.

${ }^{4}$ Magíster en Administración en Salud. Docente Universidad de Caldas, Manizales, Colombia. 


\section{TRANSCULTURAL ADAPTATION OF TEXT MESSAGES FOR SELF-CARE IN PREGNANT WOMEN}

Consuelo Vélez-Álvarez' ${ }^{1}$ Claudia Patricia Jaramillo-Ángel², Jorge Alejandro García-Ramírez ${ }^{3}$, Camilo Barrera-Valencia ${ }^{4}$

\section{Abstract}

The objetive of this work was to adapt Cross-Culturally text messages aimed at pregnant women and their companions to give tools to the academy in educational processes. Cultural adaptation was performed in 4 phases: the first phase was the review of texts translated into Spanish by researchers; then peer review; the third phase was the execution of focus groups with pregnant women and their companions and finally the development of new messages according to guidelines and standards of health care. A total of 170 messages were reviewed in the first phase; the experts reviewed 107 aimed at pregnant women, 37 to companions and 18 related with newborn care. Adjustments were made upon these messages. The result of the focus groups consolidated the process of comprehension of the messages. The article proposes a methodology for cultural adaptation that allows the academy to get messages that pregnant women need to strengthen their self-care. 


\section{ADAPTAÇÃO TRANSCULTURAL DAS MENSAGENS DE TEXTO PARA 0 AUTOCUIDADO EM GRAVIDAS}

Consuelo Vélez-Álvarez, Claudia Patricia Jaramillo-Ángel, Jorge Alejandro García-Ramírez, Camilo Barrera-Valencia

\section{Resumo}

Objetivo: adaptar transcultural-mente as mensagens de texto para gravidas e seus companheiros para usar uma ferramenta académica nos processos educativos. Realizou-se adaptações transcultural em quatro fases: a primeira partiu na revisão da literatura traduzidos ao idioma espanhol pelos professores pesquisadores, depois a segunda atividade foi o processo de revisão por expertos na área; a terceira fase seleciona por meio de grupos focais com gravidas e companheiros, a última fase a elaboração de novas mensagens de acordo a guias e normas de atenção em saúde. No total, foram revisadas 170 mensagens na primeira fase: os expertos revisarão 107 textos para gravidas, 37 para seus companheiros, 18 sobre o recém-nascido, além disso o grupo focal consolidado o processo com facilidade das mensagens. $\mathrm{O}$ artigo propõe uma metodologia para a adaptação transcultural que permite a academia obter as mensagens que as gravides precisam para melhorar seu autocuidado. 


\section{Introducción}

La salud materna es una prioridad mundial. A pesar de que este problema hizo parte de los Objetivos de Desarrollo del Milenio (ODM) para el año 2015, Colombia no logró la meta trazada de reducir la tasa de mortalidad materna (TMM) en tres cuartas partes, logrando un resultado de 55 por cada 100000 nacidos vivos, en comparación de la meta de 45 muertes maternas por 100000 nacimientos vivos (1).

La muerte de una madre se presenta como consecuencia de situaciones biológicas y socioculturales donde influye el respeto a los derechos de la mujer, a la vida, a la salud, a la información científica de calidad y a la igualdad (2). Hombres y mujeres tienen el derecho a recibir información científica oportuna y suficiente, con el fin de promover autoconocimiento, autoestima y equidad para la toma decisiones asertivas y responsables en el ejercicio de los derechos sexuales y reproductivos.

Sin embargo, la brecha en la cobertura, calidad y pertinencia de los servicios de salud sexual y reproductiva se refleja en las disparidades de acceso, por ejemplo, al control prenatal que se presenta entre poblaciones con diferencias sociales y económicas. Las mujeres que no cuentan con educación y son de escasos recursos no reciben el mismo nivel de atención prenatal o información sobre complicaciones de la gestación (3).

Se podría inferir que estrategias dirigidas a mejorar la educación en salud de las mujeres en embarazo tendrían un efecto

en el cuidado materno prenatal. Aquí se abre una oportunidad para integrar los sectores de salud y tecnología, para facilitar la comunicación y la educación de los pacientes.

La incorporación de las TIC en el campo de la salud hace referencia a la salud móvil o e-salud, la cual se consolida como un medio innovador para mejorar el acceso y la prestación de los servicios de salud, por ejemplo, a través de su teléfono móvil $(4,5)$. En este sentido, los servicios de mensajería de texto o short message services (SMS) se han empleado como intervenciones orientadas a la educación en salud de los pacientes. El uso de servicios de SMS a través de recordatorios, monitoreo de enfermedades o servicios educativos se ha asociado con mejoría en el control de enfermedades de una manera conveniente y costo- efectiva (6-9).

En el campo de la salud materna, las intervenciones han tenido como objetivo estimular una conducta de autocuidado (10-14). En el mundo existen experiencias como Text4baby o la Alianza Móvil para la acción Materna (MAMA) en Estados Unidos. Primeros ensayos controlados han mostrado que el programa tiene potencial de impacto en la salud materno-infantil (15-17). El programa se ha extendido a más de 750.000 mujeres en naciones como Sudáfrica, Bangladesh e India, adaptando culturalmente los mensajes a los idiomas locales (18). Sin embargo, el desarrollo de intervenciones en el escenario latinoamericano es reciente y escaso, existiendo experiencias en Argentina y Perú (19-22).

El proyecto mensajes para mamá (MeMa), con el nombre exacto de "Efecto de un programa de mensajería de texto sobre el autocuidado durante el período prenatal 
en una comunidad de mujeres gestantes y sus acompañantes Manizales 2015”, se apoyó en la experiencia de MAMA y la base de mensajes de texto construida en inglés y español con evidencia científica e implementada en países africanos y asiáticos para la ejecución de la estrategia en el escenario colombiano. Debe tenerse en cuenta que en el momento de aplicar un instrumento de investigación en un contexto diferente, la traducción simple puede generar interpretaciones erróneas debido al lenguaje o al contexto cultural de la comunidad donde se aplica. Para ello la literatura científica ha sugerido la ejecución del proceso de traducción, adaptación cultural y validación (TACV) con el objetivo de garantizar la reproducibilidad de la información en contextos diversos sin la pérdida de su sentido original (23).

Para el caso de MeMa, el equipo de investigación se apoyó en la base de datos de mensajes de texto ya traducida al español por MAMA. En consecuencia, el objetivo del presente estudio como fase del macroproyecto fue adaptar transculturalmente dicha base de datos para la búsqueda del autocuidado en gestantes y sus acompañantes en el contexto colombiano, como herramienta para la toma de decisiones desde la academia en el direccionamiento de los programas educativos.

\section{Métodos}

Se desarrolló un estudio de adaptación transcultural que buscaba en esencia adaptar mensajes de texto al contexto colombiano dirigido a mujeres gestantes y sus acompañantes partiendo de una base de datos previamente traducida al español.

Para avanzar sobre el consenso fiable de adaptación transcultural, se definieron cuatro fases en la búsqueda de obtener los mensajes finales sobre el autocuidado en gestantes, adaptados al contexto colombiano (figura 1).

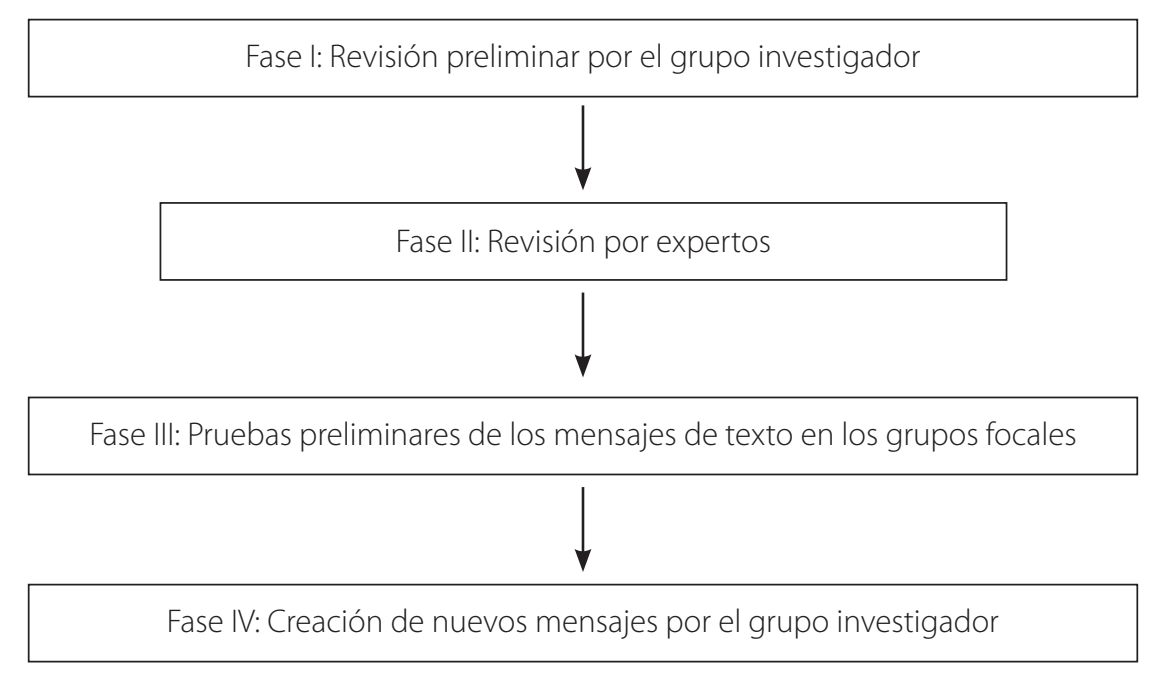

Figura 1. Esquema de la metodología utilizada para la adaptación transcultural de los mensajes de texto Fuente: elaboración propia. 
La base de datos de mensajes de texto fuente conformada por 170 mensajes fue suministrada en español por la fundación de la Naciones Unidas (United Nations Foundation) a través de su programa MAMA (Mobile Alliance for Maternal Action) para su uso libre y gratuito.

Los docentes investigadores hicieron una primera revisión de los mensajes y a través de la técnica de consenso y discusión grupal fueron efectuados algunos cambios semánticos en estos con el fin de lograr una mejor comprensión, a la luz del objetivo del proyecto y de las guías de práctica clínica actualizadas para el contexto colombiano (24).

Para la revisión por expertos fueron invitados 10 profesionales idóneos en las áreas de ginecobstetricia, pediatría, comunicaciones y medicina general con énfasis en atención primaria, de los cuales 9 efectuaron la evaluación de cada uno de los mensajes y los calificaron de acuerdo con la siguiente escala ordinal:

1. Ítem equivalente (podría utilizarse el mismo enunciado).

2. Cambios mínimos necesarios para su adaptación.

3. Cambios mayores necesarios para su adaptación.

4. Îtem no equivalente (debería excluirse).

Una vez cada experto daba su calificación podía agregar información cualitativa en el mismo instrumento relacionada con su propuesta de cambio o modificación o la justificación del por qué debía excluirse.

Tras completar la revisión por expertos los investigadores compilaron y analizaron cada uno de los conceptos dados por ellos para mejorar la adaptación de los mensajes y consolidar la base final.

De la matriz de calificación final de mensajes de texto consolidada de la revisión de expertos fueron seleccionados 30 mensajes dirigidos a gestantes y 11 mensajes dirigidos a acompañantes; como criterio de selección de estos se tuvo en cuenta aquellos mensajes que los expertos calificaron de manera más disímil y cuya comprensión requería mayor precisión por parte de la población beneficiaria. Posteriormente, para llevar a cabo la tercera de pruebas preliminares se realizaron cuatro grupos focales con mujeres gestantes y sus acompañantes para validar los mensajes seleccionados.

El trabajo en los grupos se ejecutó en alianza con instituciones privadas y públicas de la ciudad (centros comunitarios, entidades promotoras de salud - EPS- e instituciones prestadoras de servicios de salud - IPS - ). Cada grupo focal fue liderado por un moderador que hacía parte del grupo de investigación y, previo consentimiento informado, los asistentes tuvieron dos sesiones de debate: una discusión cerrada donde debieron diligenciar un formato de revisión de un conjunto de 10 mensajes de texto teniendo en cuenta los siguientes criterios de valoración de los mensajes: claridad (escribiendo la idea que comprendió del mensaje), pertinencia (muy importante, importante y poco importante) y vocabulario (definiendo si cambiaría o no alguna de las palabras del mensaje).

Posteriormente se desarrolló una discusión abierta por medio de la cual el 
moderador invitó a los participantes a expresar sus recomendaciones frente a la estrategia, así como a agregar información relacionada con mitos en el embarazo.

A partir de la moderación por uno de los investigadores, los participantes de cada grupo focal discutieron alrededor de temas como información comprendida a partir de los mensajes de texto, información adicional que desearían recibir en su embarazo, creencias y mitos durante el embarazo, y frecuencia y horarios para recibir los mensajes. La duración promedio de cada grupo focal fue entre 45 y 60 minutos.

Una vez revisada la normatividad colombiana y los resultados de los grupos focales, el grupo investigador encontró que existían algunas condiciones del período de gestación para las cuales no habían sido propuestos mensajes dirigidos a las madres y sus acompañantes. En este sentido, esta etapa consistió en proponer otros mensajes para incluir en la base de datos y consolidar la base de mensajes final para utilizar durante la intervención.

\section{Resultados}

\section{Fase I. Revisión preliminar por el grupo investigador}

La base de datos de mensajes de texto para la intervención en español fue revisada inicialmente para descartar aquellos mensajes que no aplicaran al contexto colombiano. El grupo estuvo conformado por cuatro docentes investigadores del área de la salud, según se describe en la tabla 1 .

Se revisaron un total de 170 mensajes, distribuidos así: 114 dirigidos a gestantes en su etapa prenatal, 38 dirigidos acompañantes y 18 mensajes para manejo del recién nacido y atención de la madre en el período posparto. En esta fase se excluyeron 7 mensajes de gestantes y un mensaje para acompañantes.

Tabla 1. Características de los docentes investigadores

\begin{tabular}{lll|c}
\hline \multicolumn{1}{c|}{ Trabajo actual } & \multicolumn{1}{c|}{$\begin{array}{c}\text { Calificación } \\
\text { profesoral }\end{array}$} & Especialidad & $\begin{array}{c}\text { Años de } \\
\text { experiencia }\end{array}$ \\
\hline $\begin{array}{l}\text { Decano Facultad de Ciencias para la } \\
\text { Salud Universidad de Caldas }\end{array}$ & Doctor & Salud Pública & 27 \\
$\begin{array}{l}\text { Docente Departamento de Salud } \\
\text { Pública Universidad de Caldas }\end{array}$ & Doctor & Salud Pública & 27 \\
$\begin{array}{l}\text { Docente e investigador Universidad } \\
\text { de Caldas }\end{array}$ & Magíster & $\begin{array}{l}\text { Administración } \\
\text { en Salud }\end{array}$ & 8 \\
$\begin{array}{l}\text { Docente e investigador Universidad } \\
\text { de Caldas }\end{array}$ & Médico general & & 3 \\
\hline
\end{tabular}

Fuente: Elaboración propia. 


\section{Fase II. Revisión por expertos}

Los expertos que participaron en esta fase fueron seleccionados teniendo en cuenta su especialidad y trayectoria académica relacionada con el tema (tabla 2).

Los expertos revisaron en total 162 mensajes, distribuidos de la siguiente manera: mensajes dirigidos a gestantes 107 , mensajes dirigidos a acompañantes 37 , mensajes sobre manejo de recién nacido 18 .

Aquellos mensajes que fueron calificados por 7 o más expertos bajo el criterio "Ítem equivalente (podría utilizarse el mismo enunciado)" fueron conservados en su redacción original. Los mensajes que fueron calificados por lo menos por un experto como ítem no equivalente (debería excluirse) fueron descartados de la base de datos. El resto de mensajes recibieron cambios en su redacción (tabla 3).

\section{Fase III. Pruebas preliminares de los mensajes de texto}

En los grupos focales participaron un total de 28 personas ( 20 gestantes y 8 acompañantes). El promedio de asistencia a cada grupo fue de 7 personas. En su respectivo grupo, las gestantes revisaron un conjunto de 10 de los 30 mensajes de texto y los acompañantes revisaron el grupo total de 11 mensajes de texto.

Tabla 2. Caracterización de expertos evaluadores de los mensajes de texto

\begin{tabular}{|c|c|c|}
\hline $\begin{array}{l}\text { Número } \\
\text { Experto }\end{array}$ & Institución & Cualificación profesional \\
\hline 1 & $\begin{array}{l}\text { Universidad } \\
\text { El Bosque, Colombia }\end{array}$ & $\begin{array}{l}\text { Especialidad Médica Universidad El Bosque, } \\
\text { Escuela Colombiana de Medicina, Especialización } \\
\text { The Fetal Medicine Foundation medicina fetal, } \\
\text { Ginecología y Obstetricia }\end{array}$ \\
\hline 2 & $\begin{array}{l}\text { Secretaría de Salud Públi- } \\
\text { ca, Manizales, Colombia }\end{array}$ & Psicóloga Especialista en Salud Sexual \\
\hline 3 & $\begin{array}{l}\text { Universidad del Valle, } \\
\text { Colombia }\end{array}$ & $\begin{array}{l}\text { Especialidad Médica Universidad del Valle, Especia- } \\
\text { lidad en Pediatría }\end{array}$ \\
\hline 4 & $\begin{array}{l}\text { Universidad Pontificia } \\
\text { Bolivariana, Colombia }\end{array}$ & $\begin{array}{l}\text { Maestría/Magíster Universidad Castilla la Mancha, } \\
\text { Investigación Sociosanitaria }\end{array}$ \\
\hline 5 & $\begin{array}{l}\text { Sociedad Civil, } \\
\text { Colombia }\end{array}$ & Comunicación Social \\
\hline 6 & $\begin{array}{l}\text { Universidad de } \\
\text { Caldas, Colombia }\end{array}$ & Magíster en Investigación y Tecnología Educativa \\
\hline 7 & $\begin{array}{l}\text { Universidad de La Sabana, } \\
\text { Colombia }\end{array}$ & Médica pediatra \\
\hline 8 & $\begin{array}{l}\text { Universidad de } \\
\text { Caldas, Colombia }\end{array}$ & Obstetra \\
\hline 9 & $\begin{array}{l}\text { Ministerio de Salud de } \\
\text { Perú, Perú }\end{array}$ & Investigador peruano \\
\hline
\end{tabular}

Fuente: Elaboración propia. 
Tabla 3. Calificación de los mensajes de texto por expertos

\begin{tabular}{|c|c|c|c|c|c|c|c|}
\hline \multirow{2}{*}{$\begin{array}{c}\text { Tipo } \\
\text { de mensaje }\end{array}$} & \multicolumn{2}{|c|}{ Sin modificación } & \multicolumn{2}{|c|}{$\begin{array}{l}\text { Cambios en la } \\
\text { redacción original }\end{array}$} & \multicolumn{2}{|c|}{ Excluidos } & \multirow{2}{*}{$\begin{array}{c}\text { Total } \\
\text { mensajes }\end{array}$} \\
\hline & n.o & $\%$ & n.o & $\%$ & n.o & $\%$ & \\
\hline Madres & 57 & 53 & 34 & 32 & 16 & 15 & 107 \\
\hline Acompañantes & 24 & 65 & 9 & 24 & 4 & 11 & 37 \\
\hline Recién nacido & 12 & 67 & 5 & 28 & 1 & 5 & 18 \\
\hline Total & 93 & $57 \%$ & 48 & $30 \%$ & 21 & $13 \%$ & 162 \\
\hline
\end{tabular}

Fuente: Elaboración propia.

Los resultados de la discusión cerrada los mensajes en cuanto a su claridad y con los asistentes pueden verse en la vocabulario. Ningún participante solicitó tabla 4. La mayoría de los participantes excluir alguno de los mensajes. comprendió y calificó adecuadamente

Tabla 4. Calificación de mensajes de texto en discusión cerrada

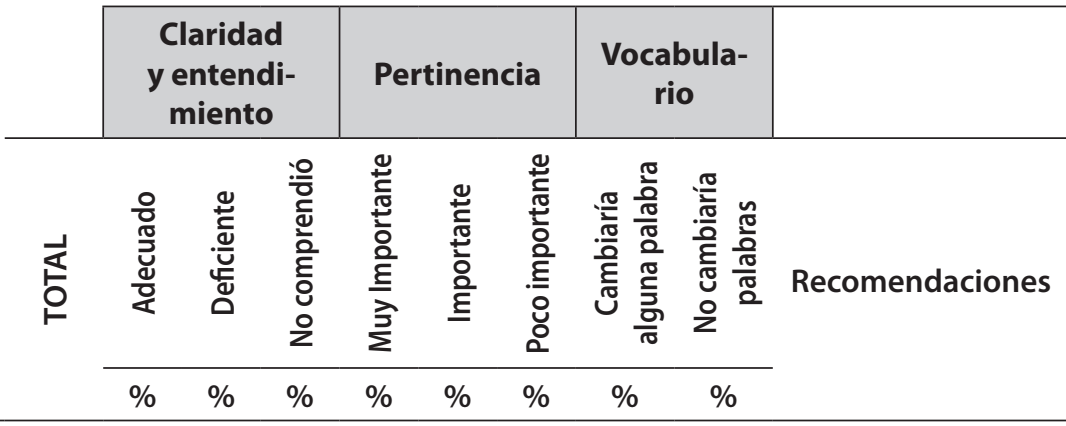

\begin{tabular}{|c|c|c|c|c|c|c|c|c|c|c|}
\hline $\begin{array}{l}\text { Gestantes } \\
\text { Grupo } 1\end{array}$ & 5 & 95 & 5 & 0 & 57 & 33 & 10 & 10 & 90 & $\begin{array}{l}\text { En el mensaje } \\
\text { cambiar la palabra } \\
\text { "ajonjolí" por la } \\
\text { expresión "arroz" }\end{array}$ \\
\hline $\begin{array}{l}\text { Gestantes } \\
\text { Grupo } 2\end{array}$ & 4 & 75 & 0 & 25 & 92 & 8 & 0 & 0 & 100 & No \\
\hline $\begin{array}{l}\text { Gestantes } \\
\text { Grupo } 3\end{array}$ & 6 & 84 & 16 & 0 & 84 & 16 & 0 & 0 & 100 & $\begin{array}{l}\text { Agregar que en el } \\
\text { embarazo debemos } \\
\text { comer mucho y } \\
\text { saludable }\end{array}$ \\
\hline $\begin{array}{l}\text { Gestantes } \\
\text { Grupo } 4\end{array}$ & 5 & 95 & 5 & 0 & 97 & 3 & 0 & 0 & 100 & No \\
\hline $\begin{array}{l}\text { Acompañan- } \\
\text { tes (Todos } \\
\text { los grupos) }\end{array}$ & 8 & 76 & 18 & 6 & 81 & 17 & 2 & 1 & 99 & No \\
\hline
\end{tabular}

Fuente: Elaboración propia. 


\section{Discusión abierta}

Sobre la comprensión de los mensajes, las mujeres expresaron por, ejemplo, que "Uno en embarazo es muy importante tomarse las pastas para el bien del bebé", "La lactancia materna es exclusiva en los primeros seis meses de vida sin complementos de fórmula", "Debo llevar una nutrición sana ybalanceada, no excederme en la alimentación", "La leche materna tiene muchas vitaminas que le pueden aportar al bebé y lo ayudan al crecimiento".

Sobre información adicional en el embarazo y la frecuencia de los mensajes de texto, las gestantes expresaron que desearían recibir los mensajes de texto durante todos los días de la semana después del almuerzo o al final del día. Además, desearían recibir más información sobre recordatorios de citas o exámenes de salud y sobre los mitos en el embarazo. Dentro de los mitos mencionados se repitieron con frecuencia los siguientes: "Una mujer que tiene la menstruación no puede cargar al bebé porque lo enferma" o que "Después del parto la mujer no puede hacer nada ni salir a la calle durante un mes" o que "hay que envolver al recién nacido como un tabaco para que no se le tuerzan los pies".

\section{Fase IV. Creación de nuevos mensajes por el grupo investigador}

Como producto de esta fase se ajustó el vocabulario de algunos mensajes que fueron propuestos a la luz de la normatividad colombiana, pero también como resultado de las observaciones hechas dentro del período prenatal (según las semanas de gestación) o del recién nacido. De igual manera, se diferenciaron los mensajes para las gestantes o para sus acompañantes (tabla 5).

\section{Discusión}

La adaptación cultural es el proceso por el cual se busca tener en cuenta las diferencias sociodemográficas y de la percepción en salud o de situaciones patológicas específicas entre la comunidad fuente y la comunidad destino para mantener la equivalencia en significado. El proceso se ha estimado en dos etapas: 1) la adaptación cultural y 2) la validación (25-26).

Según Alexandre y Guirardello para la primera etapa de adaptación cultural deben cumplirse cinco pasos: traducción directa, síntesis de traducciones, traducción inversa, consolidación por un comité de expertos y pretest (aplicabilidad y viabilidad). En esta fase se garantiza que haya una equivalencia semántica, idiomática, conceptual y experimental. Para este trabajo se partió de una base de mensajes ya traducida al español para lo cual se aplicaron los pasos cuatro y cinco.

La validación por expertos de este tipo de información es uno de los métodos utilizados para garantizar la validez de contenido que permite recolectar sistemáticamente los juicios de estos sobre un tema, procesar la información y de esta manera poder llegar a un acuerdo general $(27,28)$. Como resultados del proyecto MEMA, la revisión por el comité de expertos concluyó que el $43 \%$ de los mensajes requerían modificación o revisión 
(en vocabulario o en sentido), lo cual corrobora la necesidad de que estrategias aplicadas en contextos internacionales siempre deban adaptarse culturalmente, pues pueden presentar diferencias contextuales con la comunidad objetivo de la iniciativa en otros países.
Al respecto, autores como Guillemin plantean que en el proceso de adaptación a otra lengua se debe tener en cuenta la adaptación a la cultura y a un país, al igual que el idioma y el contexto cultural (29). En este mismo sentido, Calixto et al. (30) informan que las adaptaciones culturales exigen la adecuación de la equivalencia en la población donde va a ser aplicada.

Tabla 5. Ejemplos de mensajes de texto finales adaptados transculturalmente.

\begin{tabular}{|c|c|c|}
\hline Semana & Mensaje para gestante & Mensaje para acompañante \\
\hline 9 & $\begin{array}{l}\text { Protégete de infecciones. Lávate las manos } \\
\text { con jabón antes de preparar comidas, } \\
\text { después de ir al baño, al llegar de la calle y } \\
\text { después de tocar animales. }\end{array}$ & $\begin{array}{l}\text { Las mujeres embarazadas pue- } \\
\text { den tener cambios frecuentes de } \\
\text { ánimo. Apóyala con tu compañía y } \\
\text { comprensión. Esto le hará bien a ella } \\
\text { y al bebé. }\end{array}$ \\
\hline 11 & $\begin{array}{l}\text { En el embarazo requieres algunos exámenes } \\
\text { de sangre como VIH, hepatitis, toxoplasma } \\
\text { y síflis, para determinar cómo están tú y tu } \\
\text { bebé. El médico te orientará. }\end{array}$ & $\begin{array}{l}\text { Dile a la madre que el sangrado } \\
\text { vaginal es un signo de riesgo. Si } \\
\text { lo presenta, dale apoyo, y vayan al } \\
\text { hospital. }\end{array}$ \\
\hline 21 & $\begin{array}{l}\text { Atención: Ve a urgencias si tienes fiebre, } \\
\text { vómito, sangrado vaginal o dolor al orinar. } \\
\text { Asegúrate de que tu familia también conoz- } \\
\text { ca estos signos de emergencia. }\end{array}$ & $\begin{array}{l}\text { Atención: El embarazo hace que la } \\
\text { madre se canse. Necesita cuidados } \\
\text { extra. Ayúdala con su trabajo y dale } \\
\text { tiempo de descanso. }\end{array}$ \\
\hline 25 & $\begin{array}{l}\text { Tu bebé necesita mucho hierro. Trata de } \\
\text { comer carnes, lentejas, fríjoles, garbanzos } \\
\text { y vegetales verdes y toma tus pastillas de } \\
\text { hierro. }\end{array}$ & $\begin{array}{l}\text { Asegúrate de que la madre coma } \\
\text { carne, legumbres, frutas y vegetales } \\
\text { todos los días. Comer bien la ayuda } \\
\text { a que su bebé crezca saludable. }\end{array}$ \\
\hline 29 & $\begin{array}{l}\text { Tu bebé mide } 38 \text { centímetros desde la } \\
\text { cabecita hasta los talones. Sentirás cómo re- } \\
\text { acciona ante la luz y sonidos. Podrías sentir } \\
\text { cómo se mueve cuando le cantas. }\end{array}$ & $\begin{array}{l}\text { Ten cuidado con la hinchazón } \\
\text { repentina en las manos, pies y cara } \\
\text { de la madre. Estas son señales de } \\
\text { un problema. Llévala al hospital } \\
\text { rápido si detectas alguno de estos } \\
\text { síntomas. }\end{array}$ \\
\hline 38 & $\begin{array}{l}\text { Una sustancia gelatinosa en tu ropa interior } \\
\text { significa que el trabajo de parto empezará } \\
\text { pronto. Si tienes sangrado, como un periodo } \\
\text { menstrual, ve al hospital. }\end{array}$ & $\begin{array}{l}\text { Atención: Las contracciones fuertes } \\
\text { y regulares o romper la fuente en } \\
\text { esta edad son anormales. Lleva a la } \\
\text { madre al hospital. }\end{array}$ \\
\hline $\begin{array}{l}\text { Recién } \\
\text { Nacido }\end{array}$ & $\begin{array}{l}\text { Tu leche materna está fortaleciendo a tu } \\
\text { bebé. Aliméntalo de } 8 \text { a } 10 \text { veces por día, } \\
\text { incluyendo en la noche. No le des agua, } \\
\text { bebidas o cualquier otra cosa. }\end{array}$ & $\begin{array}{l}\text { Atención: Debes conocer los sínto- } \\
\text { mas de enfermedad del bebé: fiebre, } \\
\text { falta de respuesta, no recibe alimen- } \\
\text { to o vomita significan que necesita } \\
\text { ayuda médica. }\end{array}$ \\
\hline
\end{tabular}

Fuente: Elaboración propia. 
La fase de pretest (aplicabilidad y viabilidad) descrita en la literatura vivida en el actual proyecto en la cuarta etapa evidenció que en su mayoría la comunidad consideró los mensajes como pertinentes e importantes y no solicitaron modificaciones en su vocabulario. Se debe tener en cuenta que para esto se utilizó el método de prueba derivado de la teoría cognitiva, donde se pregunta por el significado del ítem y se solicita a la persona que lo "piense en voz alta" (think-aloud) y que reformule el enunciado con sus propias palabras para comprobar si se conservaba su significado (paraphrasing) (31).

Cabe resaltar la importancia de la evaluación cualitativa de la información la cual fue desarrollada a través de grupos focales y discusión abierta moderada por un investigador, ya que permite indagar con más profundidad los intereses de la comunidad en cuanto al sentido y objetivo de los mensajes educativos. Ejemplos de esto son las recomendaciones en cuanto a frecuencia de la comunicación, temas a reforzar, mitos, entre otros. En este sentido, autores como Barrio $(32,33)$ y Hoffman (34) señalan la importancia de mejorar el nivel de legibilidad y de contenido del material educativo e incorporar a los beneficiarios en su elaboración, porque la salud es el objetivo principal de dicho material, proceso que fue llevado a cabo con gestantes y acompañantes participantes en el estudio.

Los materiales educativos que son creados de manera efectiva pueden incidir positivamente en los hábitos de una población. Para ello se debe tener en cuenta qué información se espera recibir, proceso que implica obtener la retroalimentación de la comunidad objetivo de la iniciativa. En este sentido, el estudio demostró que las mujeres gestantes esperaban recibir información adicional sobre temas no contemplados con anterioridad. Así fue como, resultado final del desarrollo de este proceso, se crearon nuevos mensajes que complementaron la iniciativa propuesta (35).

La segunda etapa, o validación, busca garantizar que un cuestionario o instrumento preserve las propiedades psicométricas, para lo cual se aplican métodos cuantitativos de análisis para evaluar características como la fiabilidad y la validez (de contenido, de criterio, de constructo). Sin embargo, Epstain et al. reportaron que no existe un consenso internacional, ya que pueden existir hasta 31 métodos diferentes de adaptación transcultural, por lo que la elección del método corresponde también a un asunto de preferencia y logística de los investigadores, debido a que todos ellos pueden llegar al mismo resultado (36). El desarrollo de esta etapa en el presente proyecto partirá de los resultados de una intervención dirigida a una cohorte de gestantes y sus acompañantes a partir de la realización de un ensayo clínico tipo preventivo.

La adaptación cultural de los mensajes ha permitido obtener una base de datos útil, de fácil utilización y válida para nuestro entorno, aunque es importante realizar nuevos estudios que permitan valorar otras propiedades psicométricas de dicha base de datos y avanzar en la mejora de la calidad de este tipo de iniciativas. 


\section{Conclusiones}

En el proceso se buscó lograr equivalencia semántica, práctica y cultural y cubrir los principales elementos propuestos en las guías y normas de atención a las gestantes en el sistema de salud colombiano, evidenciado en quienes participaron como expertos y en las gestantes y sus acompañantes a quienes se les aplicaron las pruebas preliminares.

Los resultados de la evaluación que hicieron los expertos permitieron producir cambios en algunos mensajes, eliminar otros que a su criterio no eran pertinentes para el contexto colombiano y conservar una cifra importante de mensajes sin ningún cambio. El trabajo realizado en los grupos focales evidenció claridad en los mensajes propuestos.

Esta metodología de adaptación transcultural permite a la academia que en el momento de iniciar procesos educativos se tenga la posibilidad de contar con información que sea comprendida y aceptada por las gestantes y de esta manera se puede tener un mejor resultado en el autocuidado de éste tipo de población.

\section{Agradecimientos}

Este trabajo corresponde a la primera fase del proyecto de investigación titulado:
"Efecto de un programa de mensajería de texto sobre el autocuidado durante el período prenatal en una comunidad de mujeres gestantes y sus acompañantes Manizales 2015”, el cual fue financiado por Colciencias (Contrato No. 682 de 2014. Código: 112765741453).

\section{Declaración de conflicto de intereses:} Los autores manifiestan que no existe conflicto de intereses.

\section{Declaración de la contribución de los} autores: Consuelo Vélez-Álvarez y Claudia Patricia Jaramillo-Ángel diseñaron el estudio y analizaron los datos. Consuelo Vélez-Álvarez y Jorge Alejandro García-Ramírez redactaron la primera versión del manuscrito y estuvieron implicados en el proceso de recolección de información. Todos los autores participaron en el análisis de datos y en la revisión de la versión final del manuscrito, asimismo aprueban la versión remitida.

\section{Consideraciones éticas}

Los grupos focales fueron antecedidos por la firma del consentimiento informado. Esta investigación no presenta riesgos para los seres humanos, según la Resolución 08430 de 1993 de Colombia y la Declaración de Helsinki. Este proyecto fue aprobado por el comité de Bioética de la Facultad de Ciencias para la Salud de la Universidad de Caldas. 


\section{Referencias}

1. Programa de las Naciones Unidas para el Desarrollo (PNUD). Objetivos de Desarrollo del Milenio Informe 2015 [en línea] [Consultado 19 de mayo de 2016]. Colombia, 2015 Disponible en: http://www. co.undp.org/content/dam/colombia/docs/ ODM/undp-co-odsinformedoc-2015.pdf

2. Parra MO, Müller EÁ. Obstetricia integral siglo XXI. Bogotá: Universidad Nacional de Colombia; 2010.

3. Instituto Colombiano de Bienestar Familiar (ICBF). Módulo Conceptual Derechos Sexuales y Reproductivos. Bogotá: ICBF; 2008.

4. Cocosila M, Archer N. Adoption of mobile ICT for health promotion: an empirical investigation. Electronic Markets. 2010; 20(3-4):241-50.

5. Kwankam SY. What e-Health can offer. Bulletin of the World Health Organization. 2004; 82:800-2.

6. Free C, Phillips G, Galli L, Watson L, Felix $L$, Edwards $P$, et al. The effectiveness of mobile-health technology-based health behaviour change or disease management interventions for health care consumers: a systematic review. PLoS medicine. 2013;10(1):e1001362. Available from, DOI: 10.1371/journal.pmed.1001362

7. Vodopivec-Jamsek V, de Jongh T, Gurol-Urganci I, Atun R, Car J. Mobile phone messaging for preventive health care. The Cochrane database of systematic reviews. 2012; 12:CD007457. PubMed PMID: 23235643.

8. Krishna S, Boren SA, Balas EA. Healthcare via cell phones: a systematic review. Telemedicine journal and e-health: the official journal of the American Telemedi-
9. Riley WT, Rivera DE, Atienza AA, Nilsen W, Allison SM, Mermelstein R. Health behavior models in the age of mobile interventions: are our theories up to the task? Translational behavioral medicine. 2011 Mar; 1(1):53-71. PubMed PMID: 21796270. Pubmed Central PMCID: 3142960

10. Fjeldsoe BS, Miller YD, Marshall AL. MobileMums: a randomized controlled trial of an SMS-based physical activity intervention. Annals of behavioral medicine: a publication of the Society of Behavioral Medicine. 2010 May; 39(2):101-11. PubMed PMID: 20174902.

11. Fjeldsoe BS, Miller YD, O'Brien JL, Marshall AL. Iterative development of MobileMums: a physical activity intervention for women with young children. The international journal of behavioral nutrition and physical activity. 2012; 9:151. PubMed PMID: 23256730. Pubmed Central PMCID: 3541201.

12. Marshall AL, Miller YD, Graves N, Barnett AG, Fjeldsoe BS. Moving MobileMums forward: protocol for a larger randomized controlled trial of an improved physical activity program for women with young children. BMC public health. 2013 Jun 19; 13(1):593. PubMed PMID: 23777245. Pubmed Central PMCID: 3733692.

13. Lund S, Hemed M, Nielsen BB, Said A, Said K, Makungu MH, et al. Mobile phones as a health communication tool to improve skilled attendance at delivery in Zanzibar: a cluster-randomised controlled trial. BJOG: an international journal of obstetrics and gynaecology. 2012 Sep; 119(10):1256-64. PubMed PMID: 22805598.

14. Lund S, Nielsen BB, Hemed M, Boas $I M$, Said A, Said K, et al. Mobile phones improve antenatal care attendance in Zanzibar: a cluster randomized controlled trial. BMC pregnancy and childbirth. 2014; 14:29. PubMed PMID: 24438517. Pubmed Central PMCID: 3898378. 
15. Remick AP, Kendrick JS. Breaking new ground: the text4baby program. American journal of health promotion: AJHP. 2013 Jan-Feb; 27(3 Suppl): S4-6. PubMed PMID: 23286662.

16. Evans WD, Abroms LC, Poropatich $\mathrm{R}$, Nielsen PE, Wallace JL. Mobile health evaluation methods: the Text4baby case study. Journal of health communication. 2012;17 Suppl 1:22-9. PubMed PMID: 22548595.

17. Evans WD, Wallace JL, Snider J. Pilot evaluation of the text4baby mobile health program. BMC public health. 2012;12:1031. PubMed PMID: 23181985. Pubmed Central PMCID: 3570294.

18. United Nations Foundation. Mobile Alliance for Maternal Action, Washington2014 [citado 28 marzo de 2016]. Available from: http://http://www.mobilemamaalliance.org/.

19. Cormick G, Kim NA, Rodgers A, Gibbons L, Buekens PM, Belizan JM, et al. Interest of pregnant women in the use of SMS (short message service) text messages for the improvement of perinatal and postnatal care. Reproductive health. 2012;9:9. PubMed PMID: 22866753. Pubmed Central PMCID: 3453517.

20. Busse $P$, Curioso $W$. Diseño de mensajes de texto (SMS) para motivar a madres gestantes a que acudan a su centro de salud en una zona urbano-marginal del Perú. Lima: Instituto de Estudios Peruanos, Universidad Peruana Cayetano Heredia, 2011.

21. Universidad cayetano Heredia. Wawa Red: conetándose para una mejor salud maternainfantil en el Perú. Perú 2014. [Citado 9 de junio de 2014]. Disponible en: http://wawared.org/

22. Banco Interamericano de Desarrollo. Salud maternoinfantil en un mensaje de texto. 2014. [Citado 30 de mayo de 2014]. Disponible en: http://blogs.iadb. org/salud/2014/05/14/salud-materno-infantil-en-un-mensaje-de-texto/.

23. Ramada-Rodilla J M, Serra-Pujadas Consol, Delclós-Clanchet George L. Adaptación cultural y validación de cuestionarios de salud: revisión y recomendaciones metodológicas. Salud pública Méx [En línea]. 2013 Feb [Citado 2016 Mar 28]; 55(1):57-66. Disponible en: http://www. scielo.org.mx/scielo.php?script=sci_arttext\&pid=S0036-36342013000100009\&I$\mathrm{ng}=\mathrm{es}$

24. Ministerio de Salud y Protección Social, Colciencias. Guía de Práctica Clínica para la prevención, detección temprana y tratamiento del embarazo, parto o puerperio [en línea]. Bogotá, Colombia, 2013 [Citado 01 de abril de 2016). Disponible en: http://gpc.minsalud.gov.co/

25. Alexandre NMC, Guirardello E. Adaptación cultural de instrumentos utilizados en salud ocupacional. Rev Panam Salud Publica [Internet]. 2002 Feb [Citado 28 de marzo de 2016]; 11(2): 109111. Disponible en: http://www.scielosp. org/scielo.php?script=sci_arttext\&pi$d=S 1020-49892002000200007 \&$ Ing =en. http://dx.doi.org/10.1590/S1020-498920 02000200007.

26. Beaton DE, Bombardier C, Guillemin F, Ferraz MB. Guidelines for the process of cross-cultural adaptation of self-report measures. Spine (Phila Pa 1976). 2000 Dec 15; 25(24):3186-91. Review. PubMed PMID: 11124735.

27. García Valdés M, Suárez Marín M. El método Delphi para la consulta a expertos en la investigación científica. Rev. Cubana Salud Pública 2013; 39(2):253-267.

28. Gil-Gómez de Liaño B, Pascual-Ezama D. La metodología Delphi como técnica de estudio de la validez de contenido. Anales de Psicología 2012; 28:1011-1020. Disponible en: http://www.redalyc.org/pdf/ 167/16723774041.pdf 
29. Guillemin F. Cross-cultural Adaptation and Validation of Heatth Status Measures. Scandinavian Journal of Rheumatology. 1995; 24(2):61-3.

30. Calixto-Olalde MG, Sawada NO, Hayashida M, Mendes IAC, Trevizan MA, de Godoy S. Escala Servqual: validación en población mexicana. Texto \& Contexto Enfermagem. 2011; 20(3):326-33.

31. Willis, GB. Cognitive interviewing: $A$ tool for improving questionnaire design: a training manual. US Department of Health and Human Services, Centers for Disease Control and Prevention, National Center for Health Statistics; 1994.

32. Barrio IM, Simón P, Carmona I, Escalona I, Molina A, Ayudarte ML. Opinión de los pacientes sobre la legibilidad de los folletos de educación para la salud. Index Enferm [en línea] 2008. [Acceso 16 de abril de 2016]; 17(4). Disponible en: http:// scielo.isciii.es/scielo. php?pid=S1132$12962008000400003 \&$ script=sci_arttext

33. Barrio IM, Simón P, March J, Prieto M. Legibilidad gramatical de los prospectos de los medicamentos de más consumo y facturación en España en 2005. Rev Esp Salud Pública. 2008; 82(5):559-566.

34. Hoffmann T, McKenna K. Analysis of stroke patients and "carers" reading ability and the content and design of written materials: recommendations for improving written stroke information. Patient Education Counseling. 2006; 60(3):286-293.

35. de Oliveira SC, Lopes MV, Fernandes AF. Development and validation of an educational booklet for healthy eating during pregnancy. Rev Lat Am Enfermagem. 2014 Jul-Aug; 22(4):611-20. English, Portuguese, Spanish. PubMed PMID: 25296145.

36. Epstein J, Santo RM, Guillemin F. A review of guidelines for cross-cultural adaptation of questionnaires could not bring out a consensus. J Clin Epidemiol. 2015 Apr; 68(4):435-41. doi: 10.1016/j. jclinepi.2014.11.021. Epub 2014. Dec 17. Review. PubMed PMID: 25698408. 